\title{
A SNX10/V-ATPase pathway regulates ciliogenesis in vitro and in vivo
}

Yanqun Chen ${ }^{1,2, *}$, Bin $\mathrm{Wu}^{1,2, *}$, Liangliang $\mathrm{Xu}^{1}$, Huapeng $\mathrm{Li}^{1}$, Jianhong $\mathrm{Xia}^{1}$, Wenguang Yin ${ }^{1}$, Zhuo $\mathrm{Li}^{1}$, Dawei $\mathrm{Shi}^{1,3}$, Song $\mathrm{Li}^{4}$, Shuo $\mathrm{Lin}^{4}$, Xiaodong Shu ${ }^{1}$, Duanqing Pei ${ }^{1}$

${ }^{I}$ CAS Key Laboratory of Regenerative Biology, South China Institute for Stem Cell Biology and Regenerative Medicine, Guangzhou Institutes of Biomedicine and Health, Chinese Academy of Sciences, Guangzhou, Guangdong 510530, China; ${ }^{2}$ School of Life Sciences, University of Science and Technology of China, Hefei, Anhui 230027, China; ${ }^{3}$ School of Life Sciences, Fujian Agriculture and Forestry University, Fuzhou, Fujian 350002, China; ${ }^{4}$ Key Laboratory of Chemical Genomics, School of Chemical Biology and Biotechnology, Shenzhen Graduate School of Peking University, Shenzhen, Guangdong 518055, China

Sorting nexins (SNXs) are phosphoinositide-binding proteins implicated in the sorting of various membrane proteins in vitro, but the in vivo functions of them remain largely unknown. We reported previously that SNX10 is a unique member of the SNX family genes in that it has vacuolation activity in cells. We investigate the biological function of SNX10 by loss-of-function assay in this study and demonstrate that SNX10 is required for the formation of primary cilia in cultured cells. In zebrafish, SNX10 is involved in ciliogenesis in the Kupffer's vesicle and essential for left-right patterning of visceral organs. Mechanistically, SNX10 interacts with V-ATPase complex and targets it to the centrosome where ciliogenesis is initiated. Like SNX10, V-ATPase regulates ciliogenesis in vitro and in vivo and does so synergistically with SNX10. We further discover that SNX10 and V-ATPase regulate the ciliary trafficking of Rab8a, which is a critical regulator of ciliary membrane extension. These results identify an SNX10/V-ATPaseregulated vesicular trafficking pathway that is crucial for ciliogenesis, and reveal that SNX10/V-ATPase, through the regulation of cilia formation in various organs, play an essential role during early embryonic development.

Keywords: SNX10; V-ATPase; ciliogenesis; zebrafish

Cell Research (2012) 22:333-345. doi:10.1038/cr.2011.134; published online 16 August 2011

\section{Introduction}

Cilia are microtubule-based structures present on the cell surface, which play diverse functions during development as well as in physiological conditions [1-3]. Cilia are either motile or non-motile. Motile cilia such as the respiratory, ependymal or nodal cilia can generate fluid flows across their surfaces, which are required for several developmental and physiological processes. Nonmotile cilia of olfactory receptors or photoreceptors are involved in chemosensation and photosensation, while those of hair cells or kidney epithelia are required for

\footnotetext{
*These two authors contributed equally to this work. Correspondence: Xiaodong Shu ${ }^{\mathrm{a}}$, Duanqing Pei ${ }^{\mathrm{b}}$

${ }^{a}$ E-mail: shu_xiaodong@gibh.ac.cn

${ }^{b}$ E-mail: pei_duanqing@gibh.ac.cn

Received 21 February 2011; revised 8 May 2011; accepted 23 June 2011; published online 16 August 2011
}

mechanosensation. Furthermore, unspecialized non-motile cilia (primary cilia) are detected on almost all nondividing cells in vertebrates and they play essential roles in cell signaling such as the hedgehog or Wnt pathways [1, 4]. Because of the critical roles of cilia in normal development as well as diseased conditions, the mechanisms of cilia formation are under extensive investigations [5-7]. Ciliogenesis is tightly coupled to the cell cycle: when a cell is growth arrested, the centrosome migrates to underneath the plasma membrane and forms the basal body where the assembly of axoneme of cilium is initiated. Meanwhile, Golgi-derived vesicles form a sheath surrounding the centriole/basal body. After reaching the cell surface, the sheath fuses with the plasma membrane and forms the ciliary membrane $[8,9]$. Vesicular trafficking events are expected to be important for ciliogenesis because ciliary proteins must be transported from the Golgi to the basal body and the elongating ciliary axoneme. Genetic and biochemical studies carried out in C. rein- 
hardtii and C. elegans have provided many insights into the mechanism of the assembly of the ciliary axoneme via the intraflagellar transport (IFT)-mediated trafficking $[9,10]$. Recent studies indicate that other vesicular trafficking events are involved in ciliogenesis as well. For example, Rab8 is a regulator of vesicular trafficking and it mediates the Bardet-Biedl symdrome (BBS) protein complex regulation of ciliogenesis $[5,11]$. Fuz, a planar cell polarity effector protein, also regulates vesicular trafficking and ciliogenesis [12]. Furthermore, a recent genome-wide RNAi screen identifies proteins of the endocytic recycling pathway as modulators of ciliogenesis [13]. Despite all the progress, our understanding of the roles of vesicular trafficking during early stages of ciliogenesis remains limited.

Sorting nexins (SNXs) are PX domain-containing proteins involved in diverse intracellular processes such as endocytosis, protein sorting and degradation [14]. SNXs are present in all eukaryotes from yeast to mammals and 33 SNX family genes have been identified from the human genome. The first SNX family member, SNX1, was discovered as an EGF receptor (EGFR)-binding partner required for the lysosomal degradation of EGFR [15]. Similar intracellular functions have been reported for other SNX members ever since [14, 16]. Recently, several studies have been carried out to test the in vivo functions of selected SNX genes in the mouse model. For example, SNX1 and 2 have been knocked out and mice lacking either one of them are viable and fertile. The double knockout mice die at midgestation, which complicates the detailed analysis of the in vivo functions of SNX1 and 2 [17]. SNX13-knockout mice are also embryonic lethal [18], while SNX27 regulates the postnatal growth and survival in the mouse model [19]. So, despite the initial interests in their roles in signal transduction (mainly on the trafficking of membrane receptors), the biological functions of SNX genes remain largely unknown.

We reported previously that SNX10, when overexpressed, induces the accumulation of large vacuoles in the cytosol [20]. However, the functional implication of this observation is not clear. In this study, we perform the loss-of-function assays in cultured cells as well as the zebrafish model and demonstrate that SNX10 is required for ciliogenesis in vitro and in vivo. We further show that SNX10 binds to V-ATPase and regulates its centrosomal trafficking, which is essential for the trafficking of Rab8a vesicle to the ciliary membrane. These results reveal that the SNX10- and V-ATPase-mediated vesicular trafficking events are crucial for ciliogenesis.

\section{Results}

Zebrafish SNX10a regulates ciliogenesis in Kupffer's vesicle and left-right patterning

We first investigated the in vivo function of SNX10 in the zebrafish model. We searched the zebrafish genome with BLAST program and identified two zebrafish SNX10 homologs: dSNX10a (NM 001139462) and dSNX10b (BC154549). We tested their vacuolation activities in cultured Hela cells as described previously [20]. As shown in Figure 1A, overexpression of GFP-tagged dSNX10a induced the accumulation of large vacuoles (2.1 vacuoles/cell, $n=100$ ) while dSNX10b was not active in this assay $(0.2$ vacuoles/cell, $n=100)$. This observation suggests that dSNX10a is functionally similar to human SNX10. We then analyzed the embryonic expression pattern of zebrafish SNX10 by in situ hybridization and found that dSNX10a was not spatially restricted during early embryogenesis (Figure 1B), while dSNX10b was not detectable during the same period (data not shown). We evaluated the in vivo function of dSNX10a by performing the morpholino-mediated gene knockdown assay. We designed a translational blocking morpholino (10a-AUG) and a splice-blocking morpholino (10a-SP) that effectively induced the deletion of exon 3 from the mature RNA of dSNX10a as detected by RT-PCR and DNA sequencing (Figure 1C). The overall morphology of embryos treated with these morpholinos (the morphants) was not severely disrupted (Figure 1D), but the process of heart looping was defective in these morphants. In wild-type embryos of day 2 , the ventricle of heart is normally located to the right of the atrium ( $\mathrm{R}$ loop). However, heart looping was randomized in the dSNX10a morphants: it was either normal ( $\mathrm{R}$ loop), reversed (L loop) or failed to loop (Figure 1E and 1G). Embryos injected with a standard control morpholino (CTL-MO) or morpholinos to dSNX10b did not cause similar defect (Figure $1 \mathrm{G})$. This observation suggests that the left-right patterning process is impaired when dSNX10a is inhibited. To further confirm the left-right defect, we examined the expression pattern of southpaw (spaw, the earliest known asymmetrically expressed gene in zebrafish [21]). In control morpholino-injected embryos, spaw was asymmetrically expressed in the left lateral plate mesoderm (LPM, $91 \%, n=98)$. When dSNX10a was knocked down, spaw became bilaterally expressed in $37 \%$ of the morphants ( $n$ $=62$, Figure 1F). Thus, dSNX10a functioned upstream of spaw and could be required in a very early step of leftright patterning in zebrafish. Previous studies demonstrate that the Kupffer's vesicle (KV) is a structure essential for left-right patterning in zebrafish [22]. The beating of motile monocilia on the epithelium of $\mathrm{KV}$ generates a directional fluid flow, which is a very early asymmetrical signal in the left-right patterning cascade [23]. We tested 

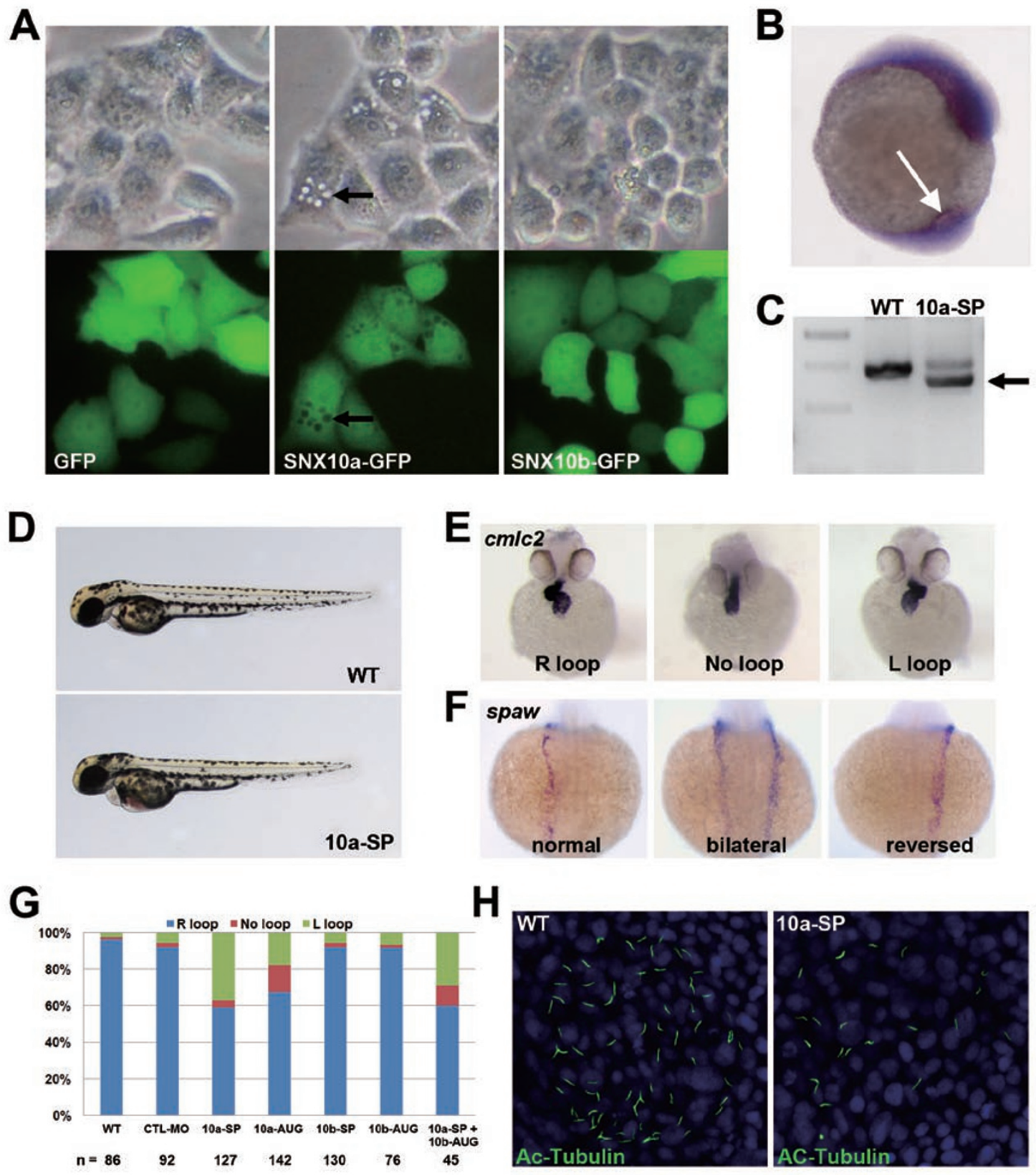

Figure 1 SNX10 regulates ciliogenesis and left-right patterning in zebrafish. (A) Overexpression of GFP-tagged zebrafish SNX10a induces the accumulation of large vacuoles (arrow) in Hela cells. SNX10b or the GFP does not have this activity. (B) The embryonic expression pattern of SNX10a at 10-somite stage as detected by in situ hybridization. It is not spatially restricted. Arrow points to the Kupffer's vesicle. (C) RT-PCR analysis for the effect of splice-blocking morpholino of SNX10a (10a-SP). DNA-sequencing analysis reveals that the exon 3 of SNX10a is deleted in the majority of the PCR products from the 10a-SP-treated embryos (the lower band). The protein product of this abnormally spliced mRNA is expected to be nonfunctional. (D) The general morphology of SNX10a morphants at day 2. SNX10a morphants show mild cardiac edema and are slightly short. (E) Heart looping visualized by in situ hybridization with the cardiac-specific marker $\mathrm{cm} / \mathrm{c} 2$ at day 2 (ventral view). In wild-type embryos, $\mathrm{R}$ loop is the predominant one. When left-right patterning is disrupted, heart looping can be reversed ( $\mathrm{L}$ loop) or remain in the midline (no loop). (F) Expression pattern of southpaw (spaw) in embryos of 16-18 somite stages (dorsal view). (G) Statistical analysis of heart looping in day 2 embryos. Heart looping is randomized in the SNX10a morphants while the standard control morpholino or morpholinos to SNX10b does not affect heart looping. (H) Ciliogenesis in KV of 10-somite stage embryos. Cilia are visualized by immunostaining with the anti-acetylated $\alpha$-tubulin antibody (green). Blue indicates the DAPI staining of nuclei. In SNX10a morphants, the average number of cilia per KV is reduced from 55 to 32. 
whether dSNX10a was required for ciliogenesis in KV. In 10-somite stage control embryos, the average number of cilia in KV was $55 \pm 8$ (from 8 embryos) with the average length of $4.2 \pm 0.7 \mu \mathrm{m}$. In 10a-SP morphants, the number of cilia per KV was reduced to $32 \pm 7$ (from seven embryos, $P=0.006$ ). The average length of cilia appeared not affected $(4.3 \pm 0.7 \mu \mathrm{m})$ (Figure $1 \mathrm{H})$. Taken together, these results demonstrated that dSNX10a was required for ciliogenesis in $\mathrm{KV}$ and left-right patterning in zebrafish.

\section{SNX10 regulates ciliogenesis in cultured cells}

We further investigated the role of SNX10 in ciliogenesis in a cell culture system. We first determined the subcellular distribution of SNX10 in the RCC10/VHL, a cell line derived from human renal carcinoma in which ciliogenesis can be easily induced by serum starvation [24]. Rab11 is a marker for pericentriolar recycling endosomes [25] and we found that SNX10 vesicles were detectable at the nearby region (Figure 2A). Similarly, when pericentriolar/centriole markers such as PCM-1, Ninein and Pericentrin were examined, we found that a fraction of SNX10 was always present around these markers (Figure $2 \mathrm{~A}$ ). When cells are serum starved, the centrosome moves to the plasma membrane where it becomes a basal body and initiates ciliogenesis. Under this condition, SNX10 was detectable around the base of the cilia (Figure 2A). The pericentriolar localization of SNX10 strongly suggests that it is involved in cilium formation. We tested this possibility by examining whether downregulation of SNX10 impaired ciliogenesis or not. We designed three independent siRNAs targeting SNX10 and all of them induced more than $80 \%$ inhibition of SNX10 at the mRNA level as determined by real-time RT-PCR (Figure 2C). A siRNA to Rab8a was used as the positive control. The RCC10/VHL cells were first treated with these siRNAs then serum starved to induce ciliogenesis. As shown in Figure $2 \mathrm{~B}$ and 2D, $49 \%$ of the control siRNA (siCTL)treated cells formed cilia $24 \mathrm{~h}$ after serum deprivation. In SNX10 siRNAs-treated cells, the ratio of ciliogenesis was reduced to $54 \%$ (si10-1), $70 \%$ (si10-2) or $34 \%$ (si10-3) of the control (siCTL) (data represent mean \pm SD from at least three independent assays, $P<0.002$ for all siRNAs to SNX10 when compared to the siCTL). Taken together, these results clearly demonstrated that SNX10 was required for cilia formation in vitro.

\section{SNX10 interacts with V-ATPase}

During our investigation of the mechanism of SNX10induced vacuolation, we found that V-ATPase was indispensible for this process. V-ATPase (vacuolar-type $\mathrm{H}^{+}$ATPase) is an ATP-driven proton pump that can induce the acidification of organelles and it is required for processes such as $\mathrm{pH}$ sensing, membrane trafficking and protein degradation $[26,27]$. V-ATPase is composed of a V1 sector (contains A, B, C, D, E, F, G and H subunits) that carries out ATP hydrolysis and a Vo sector (contains $\mathrm{a}, \mathrm{d}, \mathrm{e}, \mathrm{c}, \mathrm{c}^{\prime}$ and $\mathrm{c}^{\prime \prime}$ subunits) that is responsible for the proton pump activity. We found that the inhibition of V-ATPase blocked the accumulation of large vacuoles induced by overexpression of SNX10. As shown in Figure 3A, SNX10-GFP induced the formation of $10.0 \pm 3.3$ vacuoles per cell $(n=115)$. However, when cells were pretreated with siRNAs to the Vod1 subunit of V-ATPase, the number of vacuoles per cell was reduced to $3.7 \pm 1.8$ ( $n$ $=90, P<0.001)$. Treatment of cells with the V-ATPase inhibitor bafilomycin A1 also strongly inhibited the SNX10-induced vacuoles $(1.1 \pm 0.9$ vacuoles per cell, $n=97, P<0.001)$. The requirement of V-ATPase in the SNX10-induced vacuole formation suggests that it might co-localize with SNX10 in these vacuoles. As shown in Figure 3B, either the Vod1 or the V1D subunit of VATPase co-localized with SNX10. The distribution of V-ATPase was more restricted since there were SNX10 vesicles that were negative for V-ATPase. We next tested whether V-ATPase directly interacted with SNX10. We screened various subunits of the V-ATPase complex and found that the V1D subunit co-immunoprecipitated with SNX10. As shown in Figure 3C, when co-expressed in 293T cells, Flag-GFP-tagged V1D was able to pull down HA-tagged SNX10 (left panel) and vice versa (middle panel). We further mapped the protein domain in SNX10 that was critical for the co-immunoprecipitation with V1D. The PX domain of SNX10 alone (aa 8-125) was able to pull down V1D as efficiently as the full-length SNX10 while the C-terminal domain (CD, aa 126-202) was not required for this interaction (middle panel). SNX16, another PX domain protein, did not co-immunoprecipitate with V1D and this observation suggested that the interaction between SNX10 and V-ATPase was specific. The V1A or V1C subunit of V-ATPase did not bind to SNX10, furthermore, when they were co-expressed with the V1D, SNX10 only pulled down the V1D, but not the V1A or V1C (right panel).

\section{$V$-ATPase regulates ciliogenesis in cultured cells}

We then investigated whether V-ATPase was involved in the SNX10 regulation of ciliogenesis. We first determined the subcellular distribution of V-ATPase. When transfected into the RCC10/VHL cell, a fraction of GFP-tagged V1D co-localized with the centrosomerelated markers such as PCM-1 and Pericentrin (Figure 4A). Furthermore, under the condition when cilia were induced, V1D was detected at the base of cilia (Figure 

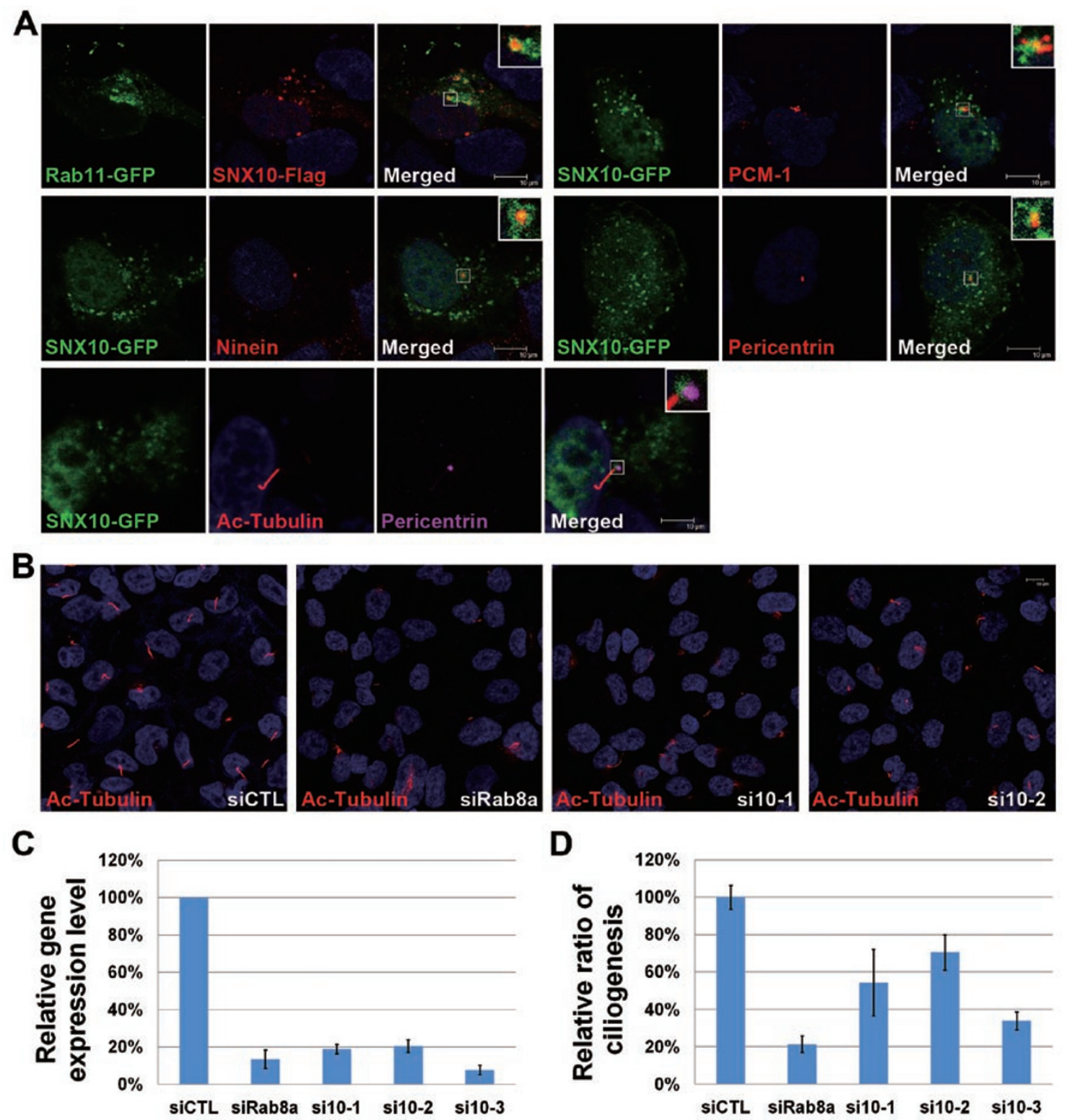

Figure 2 SNX10 regulates ciliogenesis in cultured cells. (A) Subcellular distribution of SNX10. RCC10/VHL cells are transfected with plasmid-encoding SNX10-GFP or SNX10-Flag plus Rab11-GFP. Cells are fixed $24 \mathrm{~h}$ after transfection and stained with the indicated endogenous markers: PCM-1 (pericentriolar material), Ninein or Pericentrin (centrosome). A fraction of SNX10 is detected around Rab11 (recycling endosome), PCM-1, Ninein or Pericentrin. In ciliated cells, SNX10 localizes to the base of cilia. (B) SNX10 is required for ciliogenesis in RCC10/VHL cells. Cells are transfected with a scramble control siRNA (siCTL), a positive control siRNA (siRab8a) or siRNAs to SNX10. Cilia are induced by serum starvation and visualized by immunofluorescence staining with the anti-acetylated $\alpha$-tubulin antibody (red). Nuclei are counterstained with DAPI (blue). The percentages of cells with cilia are reduced upon treatment with siRNAs to SNX10. (C) Real-time RT-PCR analysis for the efficiency of siRNAs to Rab8a and SNX10. $\beta$-actin is used as the internal control. The expression level of Rab8a or SNX10 in the siCTL-treated cells is arbitrary set as $100 \%$. More than $80 \%$ inhibition of the target gene at the mRNA level is achieved in each case. (D) Statistical analysis for B. Assays are repeated at least three times and at least 400 cells are counted for each treatment. Data represent mean \pm SD from three independent experiments $(P<0.002$ for all three siRNAs to SNX10).

4A). Similar subcellular distribution was observed for the Vod1 subunit of V-ATPase (Figures 4A and 6A). This subcellular distribution pattern of V-ATPase suggests that it could play a role during ciliogenesis. We tested this hypothesis in RCC10/VHL cells by performing siRNAmediated knockdown of V-ATPase then evaluating its 

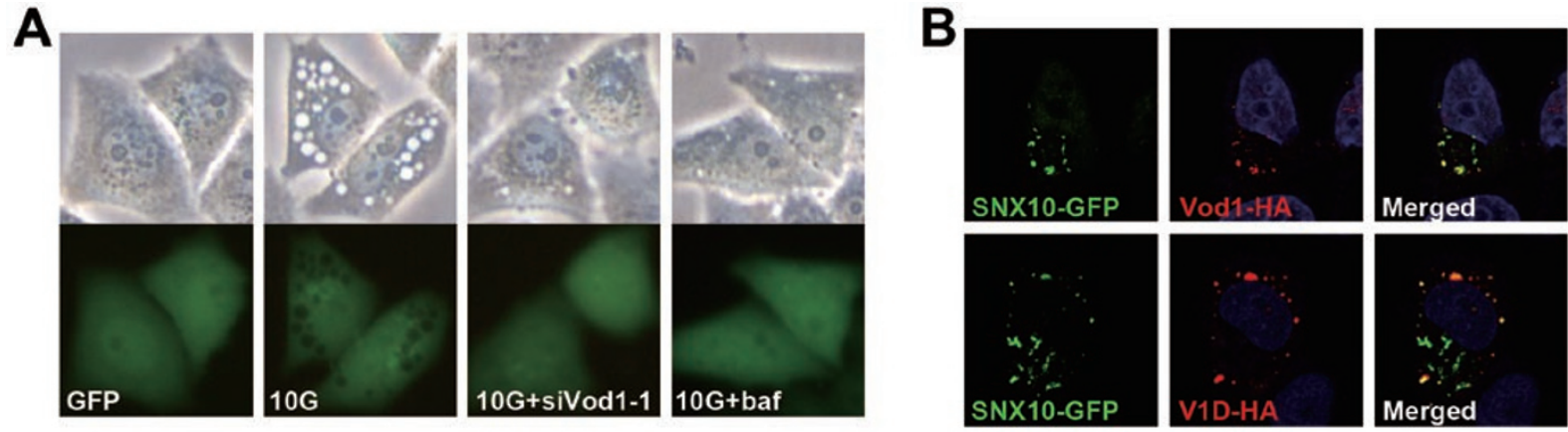
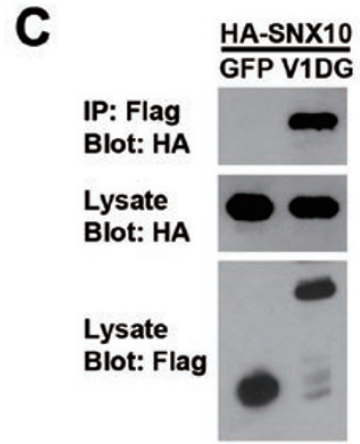

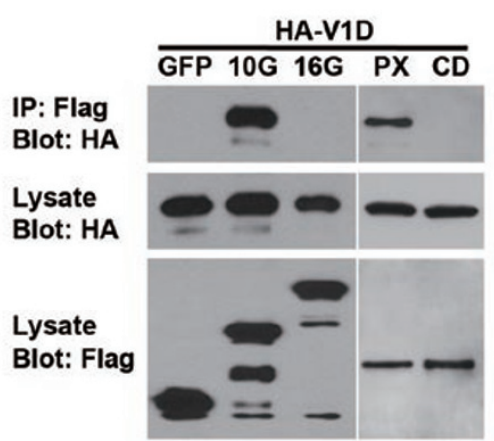

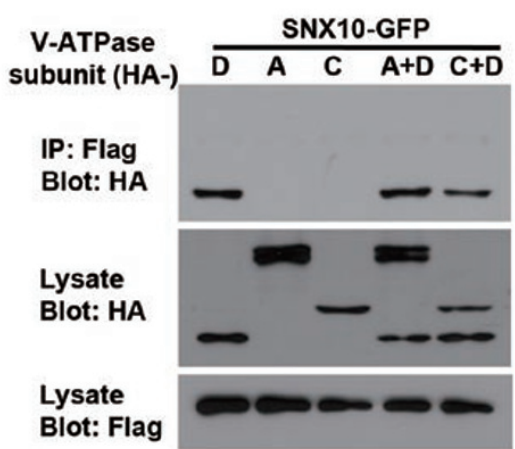

Figure 3 V-ATPase binds to SNX10 and mediates SNX10-induced vacuole formation. (A) V-ATPase is required for SNX10 to induce vacuoles in Hela cells. Pretreatment of cells with siRNA to the Vod1subunit of V-ATPase inhibits the formation of SNX10-GFP(10G)-induced vacuoles. Treatment of cells with the V-ATPase inhibitor bafilomycin A1 (baf) also inhibits the SNX10-GFP-induced vacuoles. (B) SNX10 co-localizes with V-ATPase. SNX10-GFP is co-transfected into RCC10/VHL cells with the HA-tagged Vod1 or V1D subunit of V-ATPase. Cells are fixed $24 \mathrm{~h}$ after transfection and immunostained with the mouse anti-HA antibody (red). Either subunit of the V-ATPase complex co-localizes with a fraction of SNX10-GFP. (C) SNX10 co-immunoprecipitates with the V1D subunit of V-ATPase. Left panel: HA-tagged SNX10 is co-transfected into 293T cells with the Flag-tagged GFP or Flag-GFP-tagged V1D. Cells are harvested $36 \mathrm{~h}$ after transfection and immunoprecipitation was performed with the resin-conjugated anti-Flag antibody. The levels of HA-SNX10 in cell lysates and immunoprecipitated complexes are detected by western blot using the mouse anti-HA IgG. V1D but not the Flag-GFP can pull down the HA-SNX10. Middle panel: Flag-GFP-tagged full-length SNX10 (10G) or the PX domain of SNX10 (PX, a.a. 8-125), but not the C-terminal domain of SNX10 (CD, a.a. 126-202), co-immunoprecipitates with HA-V1D. SNX16 (16G), another PX domain protein, does not pull down V1D. Right panel: HA-tagged V1A (A) and V1C (C) subunit of V-ATPase do not bind to SNX10. When they are co-expressed with V1D (D), SNX10 only pulls-down the V1D.

effect on ciliogenesis. As shown in Figure 4B, all three siRNAs induced more than $80 \%$ inhibition of V1D at the mRNA level as assayed by real-time RT-PCR. When cells were treated with these siRNAs, the ratio of cilia formation was significantly reduced (Figure 4C, $P<0.01$ when compared to the siCTL in all cases). We concluded that, similar to the SNX10, V-ATPase was required for ciliogenesis in vitro.

$V$-ATPase regulates ciliogenesis in multiple organs in zebrafish

We investigated whether V-ATPase regulated ciliogenesis in zebrafish. V-ATPase was broadly distributed during early embryogenesis as detected by in situ hybridization with a probe to the Vod1 subunit of V-ATPase
(Figure 5A). We designed translational blocking and splice-blocking morpholinos to both the Vod1 and V1D subunits of V-ATPase. Injection of embryos with these morpholinos consistently resulted in the hypopigmentation phenotype as reported [28] (Figure 5B), confirming the efficacies of these morpholinos. The heart looping was randomized in the Vod1 morphants (Figure 5C), consistent with a previous report that inhibition of $\mathrm{V}$ ATPase by chemical inhibitors induces left-right patterning defects in zebrafish [29]. We further found that SNX10 and V-ATPase functioned synergistically during left-right patterning: co-treatment of embryos with morpholinos (10a-SP and Vod1-AUG), at dosage that did not disrupt heart looping individually, effectively induced heart-looping defect (Figure 5C). Another common de- 
fect in the morphants was the formation of pronephric cysts (Figure 5D), a phenotype usually associated with cilia defect in the pronephric duct $[30,31]$. We evaluated the formation of cilia in multiple organs in V-ATPase morphants. As shown in Figure 5E, the ciliogenesis in $\mathrm{KV}$ was severely disrupted when V-ATPase was knocked down. The average number of cilia in $\mathrm{KV}$ was reduced to $27 \pm 5$ in Vod 1 morphants (from 14 embryos) or $28 \pm$ 3 in V1D morphants (from six embryos). The cilia in the pronephric duct of V-ATPase morphants were disrupted as well. These cilia in control embryos were well organized while those in Vod1 morphants were disorganized and reduced in numbers (Figure 5F). We further found that V-ATPase regulated the formation of kinocilia in hair cells. Hair cells are ciliated sensory neurons present in the inner ear and lateral line neuromasts of zebrafish [32, 33]. In V-ATPase morphants, neuromasts appeared normal (DAPI staining in Figure 5G), however, the kinocilia of hair cells were totally absent in these morphants. Taken together, these results demonstrated that V-ATPase regulated ciliogenesis in vivo.

SNX10 regulates the centrosomal distribution of $V$-ATPase

We observed that disruption of SNX10 and V-ATPase induced similar left-right patterning defects and they synergistically regulated ciliogenesis. These observations suggested that they functioned in the same genetic pathway. We further demonstrated that SNX10 co-immunoprecipitated with V-ATPase (Figure 3). Since SNX family proteins are well characterized for their roles in regulating vesicular trafficking, we hypothesized that SNX10 might regulate the subcellular trafficking of VATPase. We investigated this possibility in RCC10/VHL cells. GFP-tagged Vod1 or V1D was transfected into cells treated with siRNAs to SNX10 or a siCTL and the
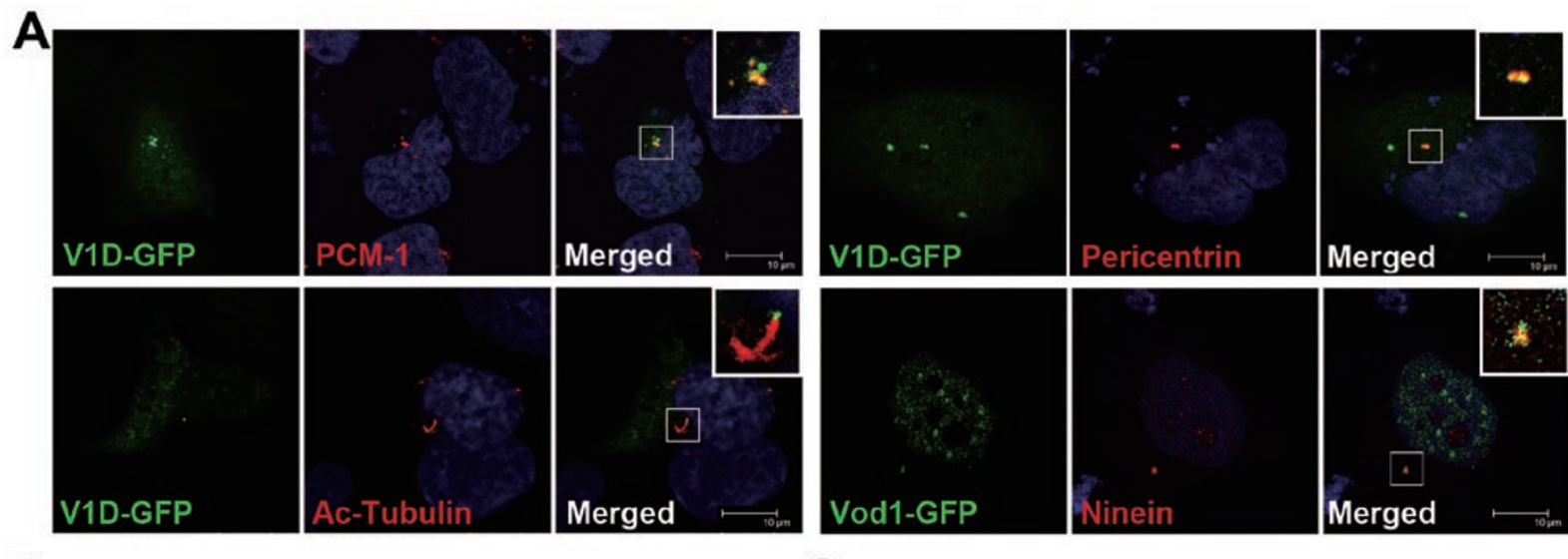

B
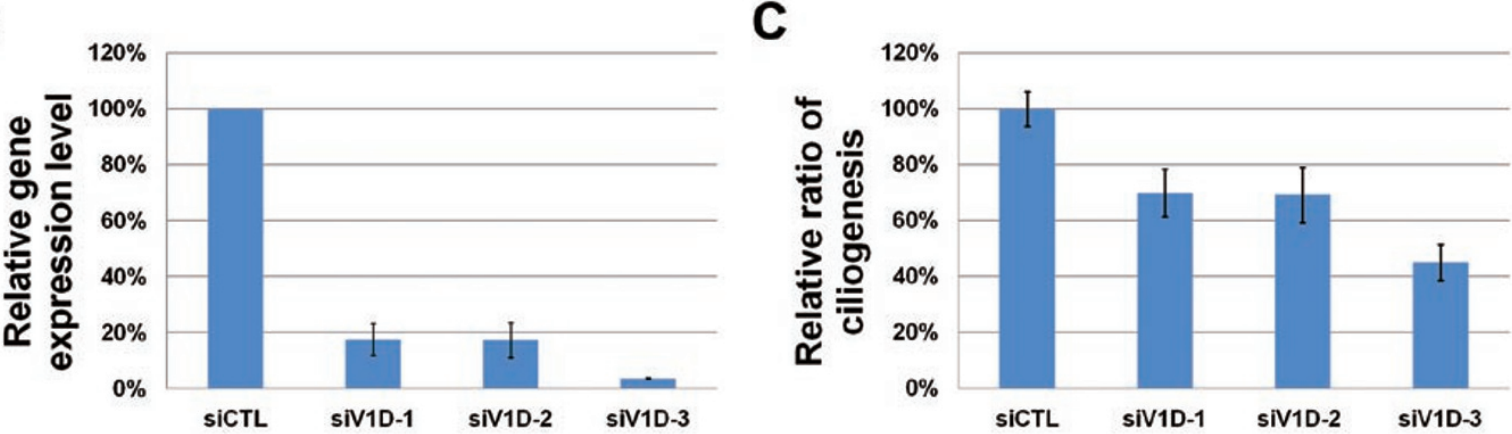

Figure 4 V-ATPase regulates ciliogenesis in vitro. (A) Subcellular distribution of V-ATPase in RCC10/VHL cells. Cells are transfected with the GFP-tagged Vod1 or V1D and immunostained for endogenous PCM-1, Pericentrin or Ninein $24 \mathrm{~h}$ after transfection. For ciliogenesis, cells are further cultured in serum-free RPMI-1640 media for $24 \mathrm{~h}$ then immunostained with the mouse anti-acetylated $\alpha$-tubulin antibody (red). V-ATPase co-localizes with PCM-1, Ninein and Pericentrin and it is present at the base of cilia. (B) Real-time RT-PCR analysis for the efficiency of siRNAs to the V1D subunit of V-ATPase. They all induce more than $80 \%$ inhibition of V1D at the mRNA level. (C) V-ATPase is required for ciliogenesis in vitro. RCC10/VHL cells are transfected with a scramble control siRNA (siCTL) or siRNAs to V1D then serum starved to induce ciliogenesis. The percentages of cells with cilia are determined as described in Figure 2. Knockdown of V1D inhibits cilia formation $(P<0.01$ for all three siRNAs). Data represent mean \pm SD from three independent experiments. 
A

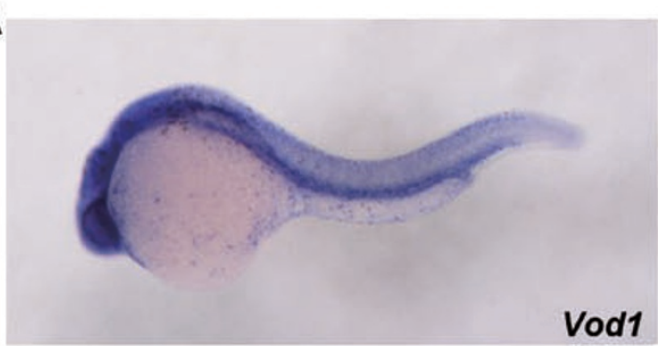

B

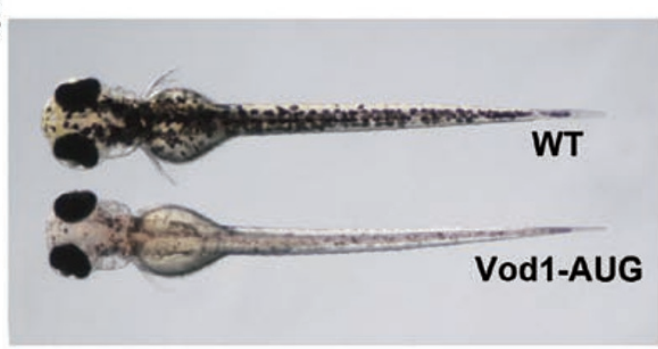

D

C
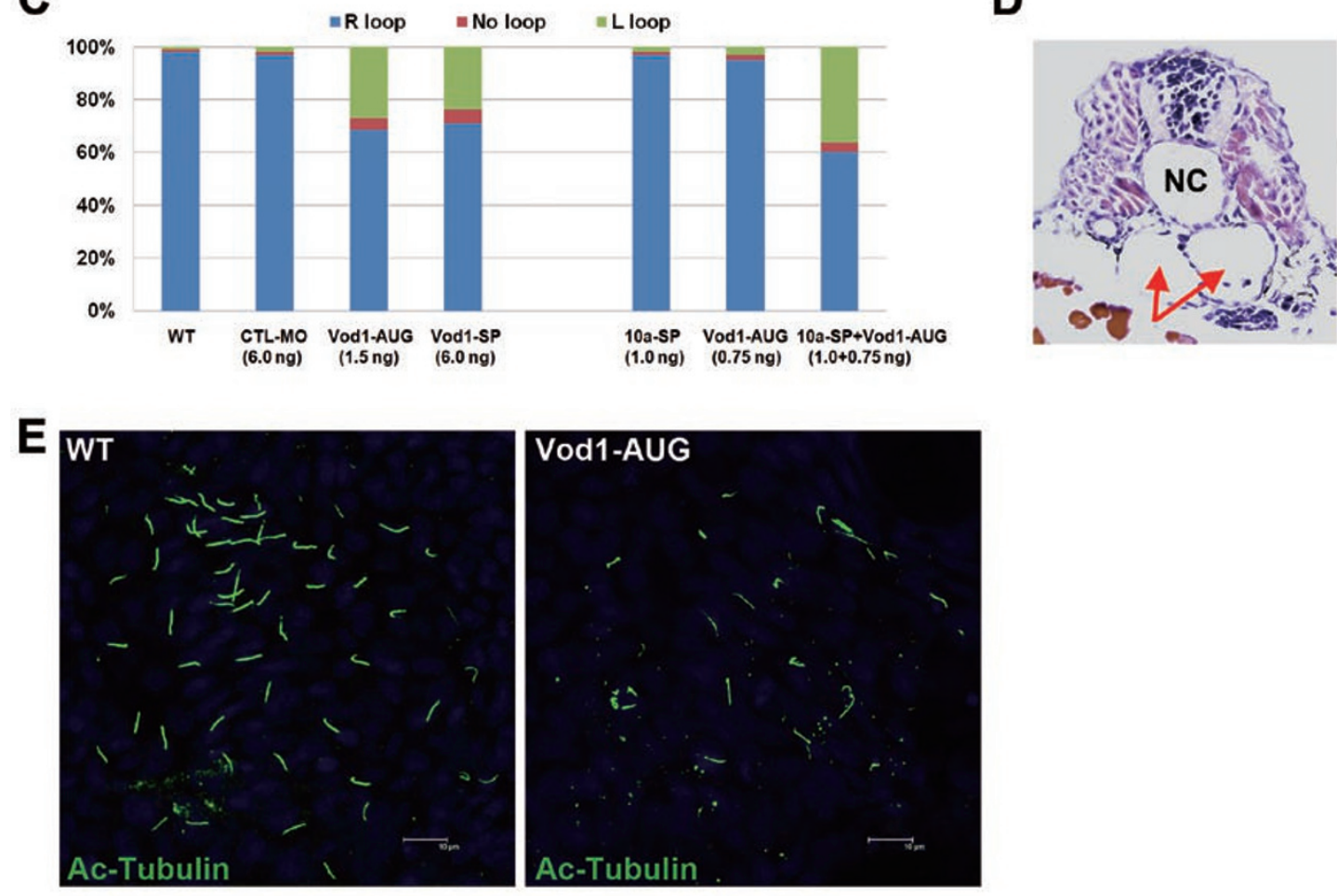

$\mathbf{F}$
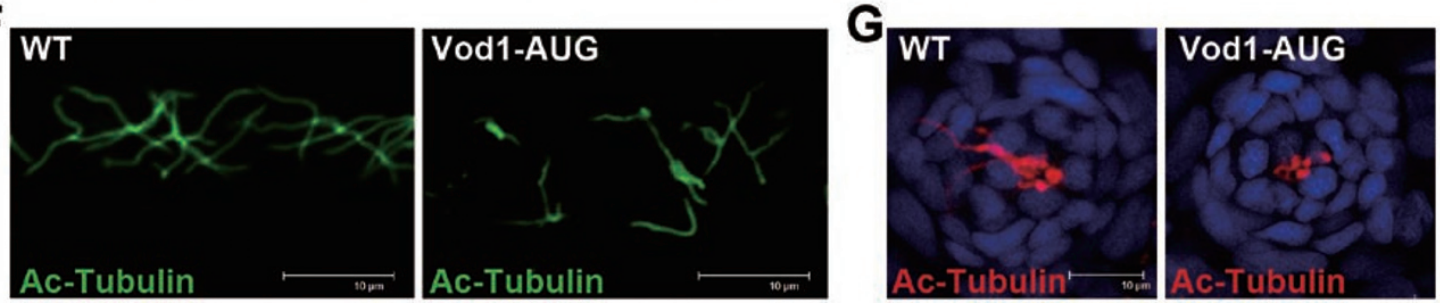

Figure 5 V-ATPase regulates ciliogenesis in multiple organs in zebrafish. (A) Embryonic expression of Vod1 at day 1 as detected by in situ hybridization. It is widely distributed. (B) Knockdown of Vod1 by morpholino impairs the pigmentation in the trunk but not the retina. Similar defect is observed in V1D morphants (data not shown). (C) Morpholino knockdown of Vod1 causes heart-looping defect similar to that in SNX10a morphants. Furthermore, Vod1 and SNX10 function synergistically in the left-right patterning of heart. 10a-SP at $1.0 \mathrm{ng} / \mathrm{embryo}$ or Vod1-AUG at $0.75 \mathrm{ng} / \mathrm{embryo}$ alone cannot effectively induce heart-looping defect, however, the combination of them is sufficient to disrupt the heart-looping process. (D) Inhibition of V-ATPase induces the formation of pronephric cyst in $65 \%$ of the treated embryos $(n=46)$. Picture shows a cross-section of Vod1 morphant at the position of the cyst (arrows). NC: notochord. (E-G) V-ATPase is involved in ciliogenesis in multiple organs in zebrafish. Cilia in KV (E, 10-somite stage), pronephric duct (F, $27 \mathrm{hpf}$ ) or hair cells of lateral line neuromasts (red staining in $\mathbf{G}$, day 2) are visualized by whole-mount fluorescence immunostaining. The nuclei are counter-stained with DAPI (blue). Ciliogenesis in the KV of V-ATPase morphants are severely inhibited. The cilia in pronephric duct are disorganized and reduced in number when V-ATPase is blocked. The neuromasts are relatively normal in the morphants (blue staining in G), but their hair cells fail to generate cilia. 
subcellular distribution of V-ATPase was evaluated. As shown in Figure 6A and 6B, a fraction of Vod1-containing vesicle co-localized with Pericentrin in $86 \%$ of the control cells. In cells pre-treated with siRNAs to SNX10, the centrosomal localization of Vod1 was reduced to $57 \%$ (for si10-1) and 51\% (for si10-2), respectively. The reduced centrosomal localization of V-ATPase after siRNA treatment was not due to the decreased protein level of Vod1. First, Vod1 vesicles at other subcellular locations were still detected after the siRNA treatment (Figure 6A). We also demonstrated that the protein level of Vod1 was not changed after siRNA treatment as measured by western blot (Figure 6C). Furthermore, the mis-localization of V-ATPase was not a result of general failure of vesicular trafficking to the centrosome, because the co-localization of $\gamma$-tubulin and Pericentrin was not disrupted by siRNAs to SNX10 (Figure 6D). Similar result was obtained when the distribution of V1D was examined (Figure 6B). We concluded that the centrosomal trafficking of V-ATPase was dependent on the activity of SNX10.

\section{SNX10 and V-ATPase regulate the ciliary trafficking of Rab8a}

Rab8a is a small GTPase involved in vesicular trafficking and it is one of the Rab family proteins that can localize to cilia $[11,34]$. Several studies have demonstrated that Rab8a is required for ciliogenesis. For example, siRNA knockdown of Rab8a inhibits ciliogenesis in hTERT-RPE cells [11] and RCC10/VHL cells (Figure 2). Rab8 is involved in ciliogenesis in vivo in zebrafish [35]. A GDP-locked variant of Rab8 fails to localize to cilia and prevents ciliogenesis, while the GTP-locked variant of Rab8 promotes the extension of both ciliary membrane and axoneme [5]. It is proposed that the Rab8apositive vesicles fuse to the outer membrane of cilia that is essential for ciliogenesis. Further studies reveal that the ciliary trafficking of Rab8a is under the regulation of BBS complex [5], CEP290 [7, 36] and PCM-1 [36].

Our finding that SNX10 partially co-localized with recycling endosome marker Rab11 (Figure 2A) prompted us to test whether SNX10 regulated the trafficking of Rab8a vesicles. When tagged Rab8a and SNX10 were transfected into the RCC10/VHL cells, SNX10 vesicles were detectable near the Rab8a around the centrosomal region (Figure $6 \mathrm{E}$ ). This observation suggested that SNX10 might be able to regulate the trafficking of Rab8a vesicles into the ciliary membrane during ciliogenesis. We tested this possibility in a RCC10/VHL cell line stably expressing Rab8a-GFP. When ciliogenesis was induced in the siCTL-treated cells by serum starvation, Rab8a-GFP localized to $43 \%$ of the cilia (Figure $6 \mathrm{~F}$ and $6 \mathrm{G})$. Consistent with Figure 2, when cells were pre-treat- ed with siRNAs to SNX10, the percentage of cells with cilia was reduced from $35 \%$ to $24 \%$. In the subset of cells that did form cilia, we analyzed the ratio of Rab8a positive cilia and found that it was reduced from $43 \%$ (siCTL) to $14 \%$ (si10-1) or $5 \%$ (si10-2). Similar results were observed when V-ATPase was inhibited by siRNAs (Figure $6 \mathrm{~F}$ and $6 \mathrm{G})$. Under these conditions, the protein level of Rab8a-GFP was not affected by the siRNA treatment (Figure $6 \mathrm{H}$ ). We proposed that the ciliary trafficking of Rab8a became defective when SNX10 or V-ATPase was inhibited. Given its regulatory role during ciliogenesis, Rab8a could, at least in part, mediate the SNX10/V-ATPase regulation of ciliogenesis.

\section{Discussion}

SNX family proteins are well characterized for their activities in regulating the endosomal sorting such as endocytosis, recycling of membrane proteins, or trafficking between various endosomes or Golgi apparatus. We reported here that SNX10 was partially distributed in the recycling endosome around the centrosomal region. SNX10 co-immunoprecipitated with V-ATPase and regulated the trafficking of V-ATPase to the centrosome. Furthermore, we showed that SNX10 was able to regulate the ciliary distribution of Rab8a. This observation revealed that SNX10 was able to regulate the intracellular trafficking of vesicles to previous unappreciated organelles such as centrosome or cilia, either directly or indirectly. It will be interesting to test whether other SNX members have similar activities.

V-ATPase pumps protons into vesicles to induce their acidification. V-ATPase-containing vesicles are distributed in diverse intracellular compartments and play critical roles during processes such as endocytosis, exocytosis, protein degradation, etc. The diverse function of V-ATPase is associated with its distinct subcellular distribution. Previous study demonstrates that the a-isoforms of the Vo sector of the V-ATPase complex are critical for the subcellular distribution of V-ATPase [27]. We found that V-ATPase could be targeted to the centrosome where ciliogenesis is initiated in a SNX10-dependent manner. This observation not only revealed new mechanism regarding the intracellular trafficking of V-ATPase, it also implied a novel biological function of V-ATPase. Indeed, we demonstrated that both the V-ATPase and SNX10 were essential for ciliogenesis in cultured cells and in zebrafish embryos.

Cilia are identified in limited cell types in invertebrates, but they are widely distributed in most of the vertebrates. Because of the critical roles of cilia in normal development and diseased conditions, the process 

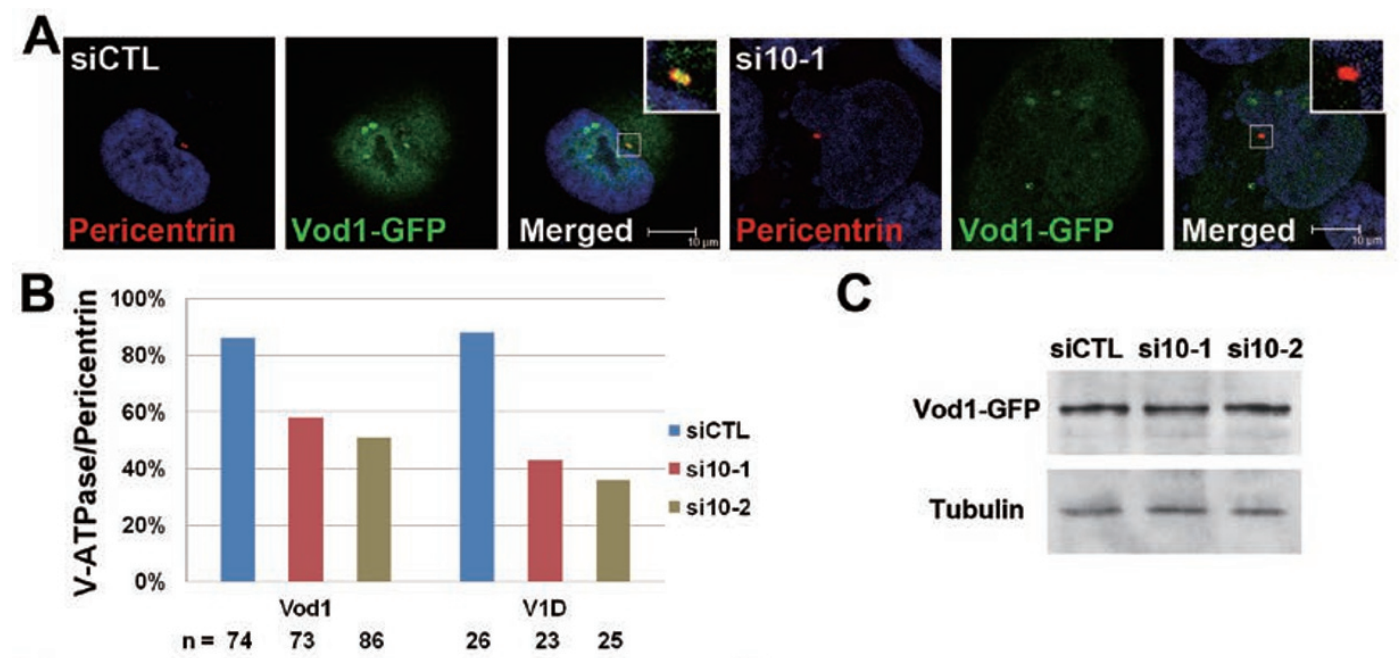

C
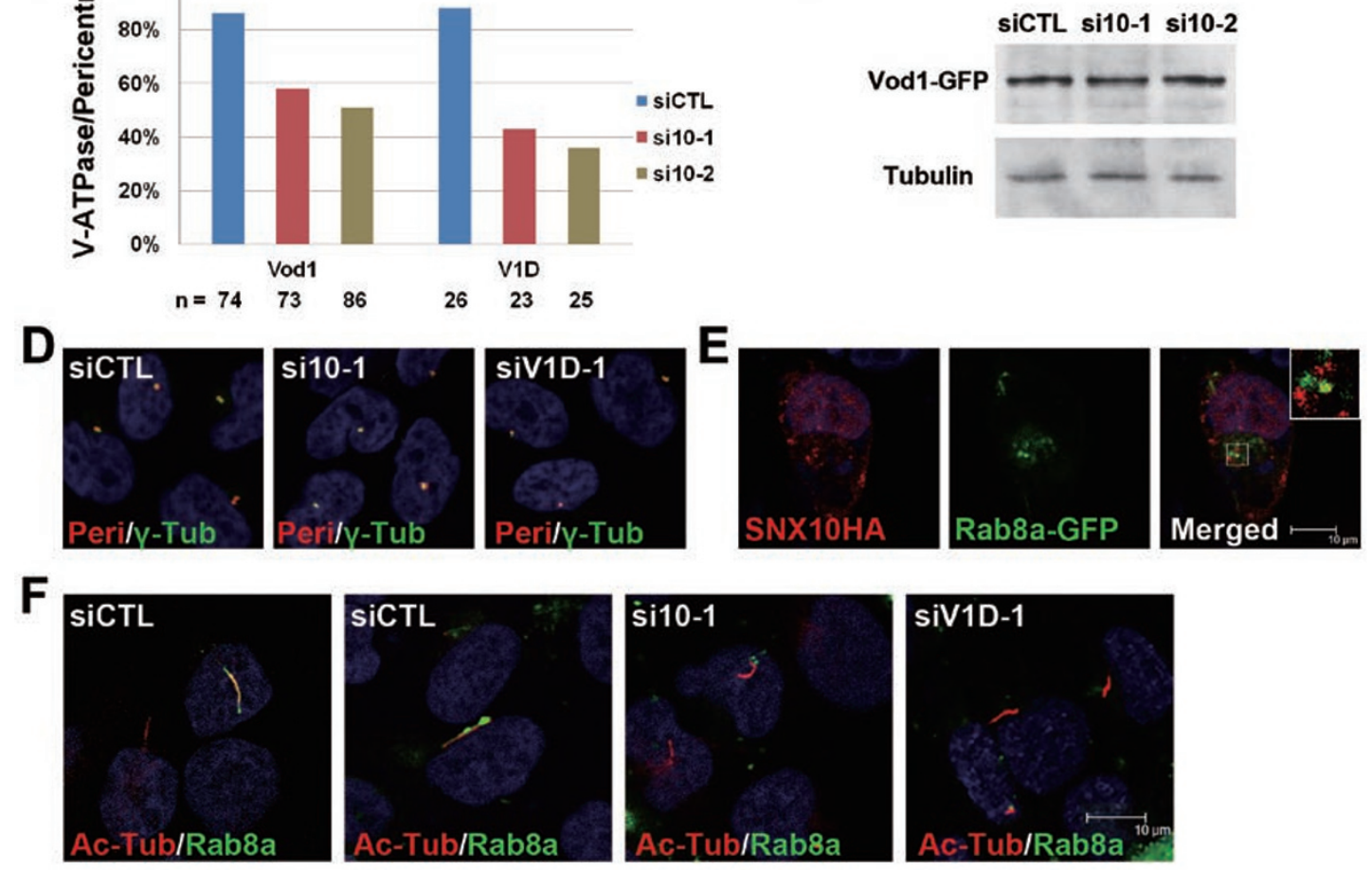

G

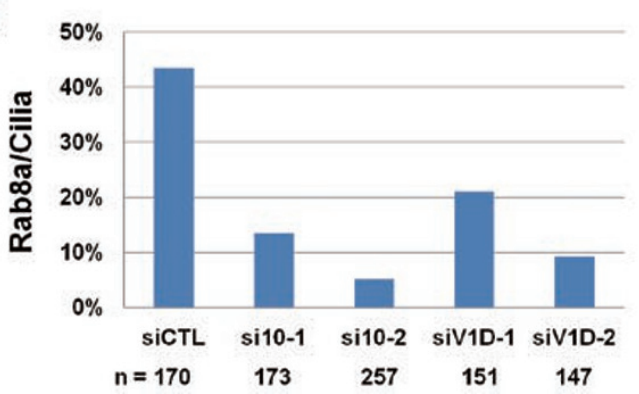

H

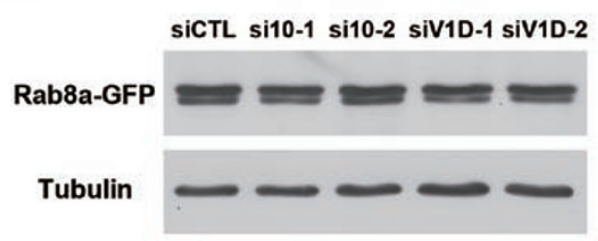

Figure 6 SNX10 regulates the intracellular trafficking of V-ATPase and Rab8a. (A) SNX10 regulates the centrosomal localization of V-ATPase. RCC10/VHL cells are first treated with siRNAs to SNX10 for $24 \mathrm{~h}$, plasmids for Vod1-GFP or V1D-GFP are then transfected into these cells. Cells are fixed and immunostained for Pericentrin after $24 \mathrm{~h}$. In control siRNA-treated cells, a fraction of Vod1-GFP co-localizes with Pericentrin (red). In SNX10 siRNAs-treated cells, the centrosomal localization of Vod1GFP is reduced. Vod1-GFP at other subcellular locations appears not affected. (B) Statistical analysis of A. (C) Western blot analysis for Vod1-GFP protein level with an anti-GFP antibody. The expression level of Vod1-GFP is not affected by the indicated siRNAs. Tubulin is the loading control. (D) Inhibition of SNX10 or V-ATPase does not affect the co-localization of Pericentrin and $\gamma$-tubulin. (E) Subcellular localization of SNX10 and Rab8a. Rab8a-GFP is enriched at the pericentriolar region and SNX10 vesicles are observed around this region. (F) SNX10 and V-ATPase regulate the ciliary trafficking of Rab8a. The Rab8a-GFP stable cell line is treated with the indicated siRNAs for $24 \mathrm{~h}$ then serum starved for another $24 \mathrm{~h}$. Cells are then fixed for immunostainig. In the control siRNA-treated cells, 43\% of the cilia is Rab8a-GFP positive. When SNX10 or V-ATPase is knocked down, in a subset of cells that form cilia, the ratio of Rab8a positive cilia is reduced. (G) Statistical analysis of $\mathbf{F}$. The ratio of Rab8a positive cilia is reduced to less than $21 \%$ in all cases when SNX10 or V-ATPase is inhibited $(P<0.05$ for all treatments when compared to the siCTL). (H) Inhibition of SNX10 or V-ATPase does not change the expression of Rab8aGFP. The protein level of Rab8a-GFP is determined by western blot with an antibody to GFP and tubulin is used as the loading control. 
of ciliogenesis has been extensively investigated in various model systems. Previous genetic screens in lower animals such as $C$. reinhardtii, $C$. elegans or D. melanogaster have identified many genes essential for cilia formation or function. More recently, proteomic analyses in mammalian cells have identified more than 2000 cilia and basal body-related proteins (Ciliaproteome V3.0, http://v3.ciliaproteome.org/). V-ATPase genes are found in all eukaryotes and SNX10 is present in all vertebrate genomes. However, both of them failed to be identified as cilia-related genes in previous studies. One possible explanation is that these genes are not the core components of ciliary structures; instead, they are regulators of vesicular trafficking temporally required for the ciliogenesis process. This is consistent with our observation that SNX10 localized to the early endosome and centrosomal regions but not the mature cilia. SNX10 regulated the subcellular distribution of V-ATPase, which also localized to the centrosome/basal body but not the cilia. We further tested several centrosome/ciliary proteins whose subcellular distributions might be under the control of SNX10 and V-ATPase and found that the ciliary entrance of Rab8a required the activity of SNX10 and V-ATPase. Rab8a is a Rab family small GTPase involved in cilia formation. When ciliogenesis is initiated, Rab8a vesicles leave the centrosome and fuse to the outer membrane of cilia. Previous studies have demonstrated that this process is under the regulation of BBS complex, CEP290 or PCM-1 $[5,7,36]$. Our results implicated that the ciliary trafficking of Rab8a was under the control of a SNX10mediated vesicular trafficking pathway. It further suggested that vesicular trafficking events other than the well-characterized IFT process also played critical roles during ciliogenesis. In conclusion, our study revealed the in vivo function of SNX10 and V-ATPase, which might provide novel opportunities to further study the mechanism of vertebrate ciliogenesis.

\section{Materials and Methods}

\section{Molecular cloning \\ Molecular cloning was performed according to standard proto- cols. The SNX10-GFP-flag and SNX10-HA constructs have been described previously [20]. The PX domain (aa 8-125) and the CD domain (aa 126-202) of SNX10 were PCR amplified using the following primers: SNX10 (8-125) forward (ATGGAGGAATTT- GTAAGTGTCTGGGTTCGAG), SNX10 (8-125) reverse (CA- GATGGCTCTGTAAGAAGAGGTGAAGGC), SNX10 (126-202) forward (ATGAATTCAGAAGACATTGAGGCGTGTGTTTC) and SNX10 (126-202) reverse (GGATTCCTGCGGAGCTGT- ATTTACTTTACATCC). The PCR products were cloned into the PCR3.1-GFP-flag vector. cDNAs for zebrafsh SNX10a and SNX10b were amplified by PCR with the following primers and cloned into the PCR3.1-GFP-flag vector: dSNX10a forward (ATG-}

GATAACACAAGCTTTGAGAAAAGGGAG), dSNX10a reverse (TATAACTGAATGGCCATGAGGCGATGAAGA) dSNX10b forward (ATGCAGGAATTTCACTGGCGTTTG) and dSNX10b reverse (CTCTGATTCACATTGCACACATTG). The ORFs of Vod1, V1D, V1A and V1C subunits of human V-ATPase were amplified from 293T cDNA and cloned into either the PCR3.1GFP-flag or the PCR3.1-HA vector. Constructs for the GFP-tagged human Rab8a and Rab11 were constructed by inserting their ORFs into the PCR3.1-nGFP vector. GFP-Rab8a was subcloned into the pRLenti vector for making the stable cell line. All constructs were confirmed by DNA sequencing and detailed information is available upon request.

\section{Cell culture, transfection and siRNA treatment}

293 T cells were cultured in DMEM (high glucose) supplemented with $10 \%$ FBS and transfection was performed using PEI reagent (polyethylenimine $1 \mathrm{mg} / \mathrm{ml}$, Sigma) [37]. RCC10/VHL cells and Hela cells were cultured in RPMI1640/10\% FBS at $37{ }^{\circ} \mathrm{C}$ with $5 \% \mathrm{CO}_{2}$. For vacuolation test, SNX10-GFP was transfected into Hela cells pre-treated with siRNAs to V-ATPase or a control siRNA using the Lipofectamine 2000 reagent according to the manufacturer's protocol (Invitrogen). For drug treatment, Bafilomycin A1 (10 nM, Sigma) was added to cells $8 \mathrm{~h}$ after transfection. Cells were analyzed $18 \mathrm{~h}$ after transfection as described [20].

Ciliogenesis was induced in RCC10/VHL cells by serum starvation. Briefly, cells were grown to confluence, washed three times with serum-free media then starved in serum-free PRMI1640 media for $24 \mathrm{~h}$ to induce ciliogenesis. DNA transfections in RCC10/VHL cells were performed using the Fugene HD transfection reagent (Roche). For transfections in 24-well plate, $1 \mu \mathrm{g}$ of plasmids in $100 \mu \mathrm{l}$ Opti-MEM were mixed with $4 \mu \mathrm{Fu}$ gene reagent. The mixture was incubated at RT for $15 \mathrm{~min}$ then added to cells $\left(5 \times 10^{5}\right)$ suspended in $500 \mu 1$ RPMI1640/10\% FBS. After incubation at RT for $30 \mathrm{~min}$, the mixture was plated into 24-well plate and cultured overnight. Stable RCC10/VHL cell line expressing GFP-Rab8a was generated by lentiviral infection using pRLenti-GFP-Rab8a [38]. siRNAs to SNX10 and V1D were designed and synthesized by Ribobio. The target sequences were as follows: siSNX10-1 (GTAATGCGTTGCTGGTACA), siSNX10-2 (CACAAGTTTGCCTTAATGA), siSNX103(CGAAGAAGATATAGAGAAT), siV1D-1 (GAAGAGTTCTATAGGTTAA), siV1D-2 (CGACAGATCCTAAAGAAGA) and siV1D-3(GGATGAAGCTATTAAGATA). sipGL4 (GATCATGAGCGGCTACGTT) was used as the negative control and siRab8a (CCATAGGAATTGACTTTAA) used as the positive control. siRNAs (final concentration of $50 \mathrm{nM}$ ) were transfected into cells using the Lipofectamine ${ }^{\mathrm{TM}}$ RNAiMAX transfection reagent (Invitrogen) according to the manufacturer's protocol. The efficiency of siRNAs was determined by real-time RT-PCR $48 \mathrm{~h}$ after transfection.

\section{Zebrafish maintenance and morpholino injection}

Zebrafish was maintained as previously described [39] and the TU line was used in this study. Morpholino antisense oligonucleotides to SNX10a, SNX10b, Vod1 and V1D genes were purchased from Gene-Tools: MO-SNX10a-AUG (TCTCAAAGCTTGTGTTATCCATCTC, 4 ng/embryo), MO-SNX10a-SP (GCAACGAGGATACTGCACTCACGTG, 2 ng/embryo), MO-SNX10b-AUG (AAACGCCAGTGAATTCCTGCATAAG, 4 ng/embryo), MO- 
SNX10b-SP (GTGGTTATTCAGACACTTACGCTTC, 2 ng/embryo), MO-ATP6VoD1-AUG (GAAAGGCATGGTGAAAAATTGGTCT, 1.5 ng/embryo), MO-ATP6VoD1-SP (AGGTCTGAAGAGCAAATTATGTAAC, 6 ng/embryo), MO-ATP6V1D-AUG (GTCGATCCGCTCTTTTCCCGACATC, 4 ng/embryo) and MO-ATP6V1D-SP (CTGAGCCCTGAAGAGACAAAACCAG, $2 \mathrm{ng} / \mathrm{embryo}$ ). The standard control morpholino (CCTCTTACCTCAGTTACAATTTATA, 4 ng/embryo) was used as the injection control. Morpholinos were injected at 1-cell stage and the treated embryos were fixed at the indicated stages for in situ hybridization [39] or whole mount immunofluorescence staining.

\section{Immunofluorescence staining}

Cells grown on glass coverslips were fixed in 4\% paraformaldehyde/PBS for $30 \mathrm{~min}$, washed with $2 \mathrm{mg} / \mathrm{ml}$ glycine in PBS for $5 \mathrm{~min}$ and permeabilized in $0.2 \%$ Triton X-100/PBS for $15 \mathrm{~min}$. After two brief washes in PBS, cells were blocked in $10 \% \mathrm{FBS} /$ PBS for $1 \mathrm{~h}$ at RT then incubated in the primary antibody either overnight at $4{ }^{\circ} \mathrm{C}$ or for $2 \mathrm{~h}$ at RT. After four washes with $1 \%$ BSA $/ 0.05 \%$ Tween-20/PBS and three washes with PBS, cells were incubated in Alex 488-, 568- or 647-conjugated goat anti-mouse $\mathrm{IgG}$ or goat anti-rabbit IgG (Molecular Probe, 1:200) secondary antibody for $1 \mathrm{~h}$. Cells were then washed four times with $1 \%$ BSA $/ 0.05 \%$ Tween-20/PBS and three times with PBS, counterstained with DAPI for 3 min (Sigma) and mounted. Primary antibodies used include rabbit anti-Ninein (Abcam, 1:100), rabbit antiPericentrin (Abcam, 1:100), mouse anti- $\gamma$-tubulin (Abcam, 1:100), mouse anti-acetylated $\alpha$-tubulin (Sigma, 1:200), mouse anti-flag (Sigma, 1:100) and mouse anti-HA (Beyotime, 1:100).

For whole-mount immunofluorescence staining, embryos at the designated stages were fixed in $4 \%$ paraformaldehyde/PBS. After several washes with PBS, embryos were treated with proteinase $\mathrm{K}$ at $37{ }^{\circ} \mathrm{C}(10 \mu \mathrm{g} / \mathrm{ml}$ for $10 \mathrm{~min}$ for 1-day-old embryos, $25 \mu \mathrm{g} / \mathrm{ml}$ for $10 \mathrm{~min}$ for 2-day-old embryos and $50 \mu \mathrm{g} / \mathrm{ml}$ for $7 \mathrm{~min}$ for 3-dayold embryos). Embryos were fixed again with 4\% PFA/PBS for 20 min. After several washes with PBS, embryos were blocked in $2 \%$ FBS/PBS and incubated with mouse anti-acetylated tubulin (Sigma, $1: 200)$ for $3 \mathrm{~h}$ at RT. After several washes with $1 \% \mathrm{BSA} / 0.05 \%$ Tween-20/PBS, embryos were incubated with Alexa Fluor 488- or 568-conjugated goat anti-mouse antibody (Invitrogen, 1:1 000) for $2 \mathrm{~h}$ at RT. Embryos were washed several times and counterstained with DAPI for $3 \mathrm{~min}$. The images were acquired using the Leica TCS-SP2 Spectral Confocal System and manipulated in Adobe Photoshop. If comparisons were to be made between images, photos were taken with identical conditions and manipulated equally.

\section{Co-immunoprecipitation and western blot}

$293 \mathrm{~T}$ cells in $60 \mathrm{~mm}$ plate were transfected with the indicated combination of plasmids using the PEI reagent. After $36 \mathrm{~h}$, cells were harvested and lysed in $600 \mu \mathrm{l}$ of TNE buffer $(20 \mathrm{mM}$ Tris$\mathrm{HCl}, \mathrm{pH} 8.0,150 \mathrm{mM} \mathrm{NaCl}, 1 \% \mathrm{NP}-40,0.5 \mathrm{mM}$ EDTA) containing $1 \mathrm{mM}$ PMSF (Sigma) and 1\% protease inhibitor cocktail (Roche). The cell lysates were centrifuged at $12000 \mathrm{rpm}$ at $4{ }^{\circ} \mathrm{C}$ for $5 \mathrm{~min}$ and the supernatants were incubated with resins conjugated to the anti-flag antibody (Sigma) at $4{ }^{\circ} \mathrm{C}$ for $4 \mathrm{~h}$ to overnight. After the incubation, the resins were washed with TNE for 10 times and then eluted with $0.5 \mathrm{mg} / \mathrm{ml}$ flag peptide (Sigma) in TNE. The eluants were cleared by centrifugation and the supernatants were boiled in SDS sample buffer. Samples were separated by
SDS-PAGE and transferred to PVDF membrane (Millipore) by electrophoresis. Western blot was performed using the standard protocol. The primary antibodies used were mouse anti-flag (Sigma, 1:5 000) and mouse anti-HA (Beyotime, 1:1 000).

\section{Acknowledgments}

We are grateful to Hanbing Zhong (Peking University, China) and Miguel Esteban (GIBH, China) for reagents and helpful discussions. We thank members of the Pei Lab for technical assistance. This work is supported by the National Natural Science Foundation of China (30630039, 30700410, 30871404 and 90813033), Ministry of Science and Technology 973 program (2009CB941102, 2009CB941203), Knowledge Innovation Program of the Chinese Academy of Sciences (KSCX2-YW-R-221, KSCX1-YW-02-1) and CAS 100-talent project (X S).

\section{References}

1 Gerdes JM, Davis EE, Katsanis N. The vertebrate primary cilium in development, homeostasis, and disease. Cell 2009; 137:32-45.

2 Satir P, Christensen ST. Overview of structure and function of mammalian cilia. Annu Rev Physiol 2007; 69:377-400.

3 Fliegauf M, Benzing T, Omran H. When cilia go bad: cilia defects and ciliopathies. Nat Rev Mol Cell Biol 2007; 8:880-893.

4 Singla V, Reiter JF. The primary cilium as the cell's antenna: signaling at a sensory organelle. Science 2006; 313:629-633.

5 Nachury MV, Loktev AV, Zhang Q, et al. A core complex of BBS proteins cooperates with the GTPase Rab8 to promote ciliary membrane biogenesis. Cell 2007; 129:1201-1213.

6 Spektor A, Tsang WY, Khoo D, Dynlacht BD. Cep97 and CP110 suppress a cilia assembly program. Cell 2007; 130:678-690.

7 Tsang WY, Bossard C, Khanna H, et al. CP110 suppresses primary cilia formation through its interaction with CEP290, a protein deficient in human ciliary disease. Dev Cell 2008; 15:187-197.

8 Sorokin S. Centrioles and the formation of rudimentary cilia by fibroblasts and smooth muscle cells. J Cell Biol 1962; 15:363-377.

9 Pedersen LB, Veland IR, Schroder JM, Christensen ST. Assembly of primary cilia. Dev Dyn 2008; 237:1993-2006.

10 Silverman MA, Leroux MR. Intraflagellar transport and the generation of dynamic, structurally and functionally diverse cilia. Trends Cell Biol 2009; 19:306-316.

11 Yoshimura S, Egerer J, Fuchs E, Haas AK, Barr FA. Functional dissection of Rab GTPases involved in primary cilium formation. J Cell Biol 2007; 178:363-369.

12 Gray RS, Abitua PB, Wlodarczyk BJ, et al. The planar cell polarity effector Fuz is essential for targeted membrane trafficking, ciliogenesis and mouse embryonic development. Nat Cell Biol 2009; 11:1225-1232.

13 Kim J, Lee JE, Heynen-Genel S, et al. Functional genomic screen for modulators of ciliogenesis and cilium length. $\mathrm{Na}$ ture 2010; 464:1048-1051.

14 Cullen PJ. Endosomal sorting and signalling: an emerging role for sorting nexins. Nat Rev Mol Cell Biol 2008; 9:574-582. 
15 Kurten RC, Cadena DL, Gill GN. Enhanced degradation of EGF receptors by a sorting nexin, SNX1. Science 1996; 272:1008-1010.

16 Seet LF, Hong W. The Phox (PX) domain proteins and membrane traffic. Biochim Biophys Acta 2006; 1761:878-896.

17 Schwarz DG, Griffin CT, Schneider EA, Yee D, Magnuson T. Genetic analysis of sorting nexins 1 and 2 reveals a redundant and essential function in mice. Mol Biol Cell 2002; 13:35883600 .

18 Zheng B, Tang T, Tang N, et al. Essential role of RGS$\mathrm{PX} 1 /$ sorting nexin 13 in mouse development and regulation of endocytosis dynamics. Proc Natl Acad Sci USA 2006; 103:16776-16781.

19 Cai L, Loo LS, Atlashkin V, Hanson BJ, Hong W. Deficiency of sorting nexin 27 (SNX27) leads to growth retardation and elevated levels of N-methyl-D-aspartate receptor 2C (NR2C). Mol Cell Biol 2011; 31:1734-1747.

20 Qin B, He M, Chen X, Pei D. Sorting nexin 10 induces giant vacuoles in mammalian cells. J Biol Chem 2006; 281:3689136896.

21 Long S, Ahmad N, Rebagliati M. The zebrafish nodal-related gene southpaw is required for visceral and diencephalic leftright asymmetry. Development 2003; 130:2303-2316.

22 Essner JJ, Vogan KJ, Wagner MK, Tabin CJ, Yost HJ, Brueckner M. Conserved function for embryonic nodal cilia. Nature 2002; 418:37-38.

23 Essner JJ, Amack JD, Nyholm MK, Harris EB, Yost HJ. Kupffer's vesicle is a ciliated organ of asymmetry in the zebrafish embryo that initiates left-right development of the brain, heart and gut. Development 2005; 132:1247-1260.

24 Esteban MA, Harten SK, Tran MG, Maxwell PH. Formation of primary cilia in the renal epithelium is regulated by the von Hippel-Lindau tumor suppressor protein. J Am Soc Nephrol 2006; 17:1801-1806.

25 Ullrich O, Reinsch S, Urbe S, Zerial M, Parton RG. Rab11 regulates recycling through the pericentriolar recycling endosome. J Cell Biol 1996; 135:913-924.

26 Forgac M. Vacuolar ATPases: rotary proton pumps in physiology and pathophysiology. Nat Rev Mol Cell Biol 2007; 8:917929.

27 Marshansky V, Futai M. The V-type H+-ATPase in vesicular trafficking: targeting, regulation and function. Curr Opin Cell Biol 2008; 20:415-426.

28 Nuckels RJ, Ng A, Darland T, Gross JM. The vacuolarATPase complex regulates retinoblast proliferation and survival, photoreceptor morphogenesis, and pigmentation in the zebrafish eye. Invest Ophthalmol Vis Sci 2009; 50:893-905.

29 Adams DS, Robinson KR, Fukumoto T, et al. Early, H+-VATPase-dependent proton flux is necessary for consistent leftright patterning of non-mammalian vertebrates. Development 2006; 133:1657-1671.

30 Drummond IA, Majumdar A, Hentschel H, et al. Early development of the zebrafish pronephros and analysis of mutations affecting pronephric function. Development 1998; 125:46554667.

31 Sun Z, Amsterdam A, Pazour GJ, Cole DG, Miller MS, Hopkins N. A genetic screen in zebrafish identifies cilia genes as a principal cause of cystic kidney. Development 2004; 131:4085-4093.

32 Nicolson T. The genetics of hearing and balance in zebrafish. Annu Rev Genet 2005; 39:9-22.

33 Edge AS, Chen ZY. Hair cell regeneration. Curr Opin Neurobiol 2008; 18:377-382.

34 Li Y, Hu J. Small GTPases and cilia. Protein Cell 2011; 2:13-25.

35 Omori Y, Zhao C, Saras A, et al. Elipsa is an early determinant of ciliogenesis that links the IFT particle to membraneassociated small GTPase Rab8. Nat Cell Biol 2008; 10:437444.

36 Kim J, Krishnaswami SR, Gleeson JG. CEP290 interacts with the centriolar satellite component PCM-1 and is required for Rab8 localization to the primary cilium. Hum Mol Genet 2008; 17:3796-3805.

37 Bieber T, Elsasser HP. Preparation of a low molecular weight polyethylenimine for efficient cell transfection. Biotechniques 2001; 30:74-77, 80-81.

38 Nifontova IN, Sats NV, Surin VL, Svinareva DA, Gasparian ME, Drize NJ. Infection of stromal and hemopoietic precursor cells with lentivirus vector in vivo and in vitro. Bull Exp Biol Med 2008; 145:133-136.

39 Westerfield M. The Zebrafish Book. A Guide for the Laboratory Use of Zebrafish (Danio rerio). Eugene: University of Oregon, 1995. 